\title{
EXPRESSION OF TUMOR ASSOSIATED AND EPITHELIAL-MESENCHYMAL TRANSITION MARKERS IN 2D AND 3D CELL CULTURES OF MCF-7
}

\author{
Tetiana Herheliuk \\ Department of Biotechnical Problems of Diagnostics \\ Institute for Problems of Cryobiology and Cryomedicine National Academy of Science of Ukraine \\ 42/1 Nauky ave., Kyiv, Ukraine, 03028 \\ gergelyuk87@mail.ru \\ Olena Perepelytsina \\ Department of Biotechnical Problems of Diagnostics \\ Institute for Problems of Cryobiology and Cryomedicine National Academy of Science of Ukraine \\ 42/1 Nauky ave., Kyiv, Ukraine, 03028 \\ olenaquail@rambler.ru \\ Natalia Yurchenko \\ RE Kavetsky Institute of Experimental Pathology \\ Oncology and Radiobiology \\ National Academy of Science of Ukraine \\ 45 Vasylkivska str., Kyiv, Ukraine, 03022 \\ kostyason1995@rambler.ru \\ Mykhailo Sydorenko \\ Department of Biotechnical Problems of Diagnostics \\ Institute for Problems of Cryobiology and Cryomedicine National Academy of Science of Ukraine \\ 42/1 Nauky ave., Kyiv, Ukraine, 03028 \\ olenaquail@gmail.com \\ Lyudmila Ostapchenko \\ Educational and Scientific Centre "Institute of Biology \& Medicine" \\ 2 Glushkova ave., Kyiv, Ukraine, 03187
}

\begin{abstract}
The target effects on the expression of epithelial-mesenchymal transition regulation molecules are promising for cancer therapy, including breast cancer. 3D cell culture is a model for studying epithelial-mesenchymal transition in vitro and may become a test system for anticancer therapy.

Aim of research. The aim of this research was to evaluate and compare the expression of tumor associated and epithelial-mesenchymal transition markers in tumor cells of breast adenocarcinoma (MCF-7 cell line) in 2D and 3D cell culture.

Methods. For realization of the aim MCF-7 cell line (breast adenocarcinoma) was chosen as an experimental model in vitro. The monolayer cell culture was cultured in standard conditions $\left(37^{\circ} \mathrm{C}, 5 \% \mathrm{CO}_{2}\right.$, humidity $\left.95 \%\right)$. The initial density of inoculated cells was $2 \times 10^{4}$ cells $/ \mathrm{cm}^{2}$. The cells were incubated for two days before their use in the experiment. For the initial generation of spheroids the monolayer cell culture was removed off the substrate after the four days of incubation, using $0,25 \%$ Trypsin-EDTA, and placed in nutrient medium with $5 \%$ carboxymethyl cellulose (Bio-Rad, USA) at concentration of $5 \times 10^{5} \mathrm{cells} / \mathrm{ml}$. Then the plates were incubated on an orbital shaker (Orbital shaker, PSU-10i, Biosan, Latvia) at $50 \mathrm{rpm}$ for 3-5 hours. Half of culture medium was replenished every 3 days. A spheroid culture was maintained for 14 days. Detection of markers (ER, p53, EpCAM, vim, AE1/AE3, panCK, EGFR) in 2D and $3 \mathrm{D}$ cell culture was performed using immunohistochemistry method with primary monoclonal antibodies. Histological samples of cells were photographed to compare the morphological characteristics and the expression of proteins in monolayer and spheroid culture

Results. The results demonstrated that the percentage of tumor marker positive cells $(\mathrm{ER}+, \mathrm{EGFR}+$, EpCAM+, panCK+, $\mathrm{AE} 1 / \mathrm{AE} 3+)$ in monolayer culture is 1.25-2 times than more in spheroid culture. In contrast, tumor spheroids consist of fewer cells with the expression of epithelial markers such as EpCAM and AE1/AE3, but they contain a large number of cells that expressed mesenchymal marker vimentin by $5 \%$ and p53 by $10 \%$. This may indicate that the cells acquire a mesenchymal phenotype. However, tumor cells of monolayer cell culture were not expressed vimentin.
\end{abstract}


Conclusions. Our results demonstrated the differences of expression of tumor associated and epithelial-mesenchymal transition markers in 2D and 3D breast cancer cell cultures. Thus, the percentage of epithelial markers (Cytokeratines and epithelial cell adhesion molecule) in tumor spheroids is less than in cells of monolayer however spheroids cells begin expressing a mesenchymal marker - vimentin. In 3D cell culture only the outer cell layers expressed tumor associated proteins unlike 2D cell culture in which all of cells showed equally expression. Reduced of manifestation of tumor associated markers in 3D cell culture may indicate an increase of stem properties. These data showed that $3 \mathrm{D}$ cell culture more than $2 \mathrm{D}$ cell culture characterized processes of epithelialmesenchymal transition.

Keywords: multicellular tumor spheroids, 2D and 3D cell culture, epithelial-mesenchymal transition, breast cancer, MCF-7.

\section{Introduction}

Cancer cell lines are widely used in cancer development studies and for testing of the anticancer drugs. Recent studies have shown that the most adequate model of the tumor population in vitro is a multilayer (3D) cell culture (multicellular tumor spheroids, MCTS) [1]. MCTS model is helpful for understanding of disease development mechanisms, invasion, metastasis and resistance to anticancer therapy [2]. In the tissue in vivo tumor cells have strong connections with neighboring cells and the extracellular matrix. Also, the 3D cell cultures are useful for evaluating the impact of cell-cell interactions on tumor development in vitro. Tumor cells in 3D cultures actively interact with each other, with the extracellular matrix and microenvironment. These interactions affect the proliferation, differentiation, cell morphology, gene expression and protein synthesis. The structure of MCTS is similar to the micro metastases or tumor at early avascular stage. Furthermore, 3D spheroids are composed of cells at different stages of development and under influence of various factors (proliferating, dormant, apoptotic and necrotic cells) [3]. The most outer layers of spheroid consist of actively proliferating cells, but core contains of dormant or necrotic cells, because these cells receive less oxygen, growth factors and nutrients. Other differences between 2D and $3 \mathrm{D}$ cell cultures are gene expression level, proliferation rate, migration ability and resistance to anticancer therapy [4]. Unlike monolayer, cells of spheroid show less ability to apoptosis, which is caused by radio- or chemotherapy, due to the reduction or blocking of cell death receptor expression [5]. 3D cell culture spatial architecture plays an important role in the testing of anticancer drugs therapeutic effect, and in evaluation of the invasive capacity of the transformed cells.

In addition, we suppose that the study of changes in the phenotype of tumor cells from epithelial to mesenchymal in MCTS is very relevant. The process by which epithelial cells lose their cell polarity and cell-cell adhesion, and gain migratory and invasive properties to become mesenchymal stem cells is called epithelial-mesenchymal transitions (EMT) and is the object of primary interest for the development of anti-tumor therapy. EMT is a key process for embryonic development, as well as tissue regeneration and tumor progression process in an adult organism. Tumor cells that are exposed to EMT can inhibit antitumor mechanisms of the organism, migrate, do not respond to apoptosis, and do not react to the action of anticancer drugs. These cells act as a reservoir which replenishes the tumor cell population [6]. EMT phenomenon explained the reasons for the formation of distant metastases of tumors of epithelial origin, including breast cancer (BC). Features of EMT are the destruction of cell-cell contacts, which leads to the loss of epithelial characteristics and formation of mesenchymal morphology [7]. EMT-cells actively proliferate and self-renew, resulting in increased amount of heterogeneous populations. As a result, cell motility increased and metastatic colonies in distant places are formed [8]. Thus, the target effect on the expression of molecules that regulate EMT is promising for cancer therapy, including breast cancer.

\section{Aim of research}

The aim of this study was to evaluate and compare the expression of major EMT markers in tumor cells of breast adenocarcinoma (MCF-7 cell line) in 2D and 3D cell culture. 


\section{Materials and methods}

An MCF-7 cell line (breast adenocarcinoma) was kindly offered by the bank of Cell Lines from Human and Animal Tissues, Kavetsky` Institute of Experimental Pathology, Oncology and radiobiology, National Academy of Science of Ukraine. The monolayer cell culture was cultured in standard conditions $\left(37^{\circ} \mathrm{C}, 5 \% \mathrm{CO}_{2}\right.$, humidity $95 \%$ ), using DMEM nutrient medium (Dulbecco's Modified Eagle Medium, "Sigma», USA) with $10 \%$ fetal serum (Sigma, USA), 2 mM L-Glutamine (Sigma, USA), and $40 \mathrm{mg}$ Gentamicin (Biopharma, Ukraine). The initial density of inoculated cells was $2 \times 10^{4}$ cells $/ \mathrm{cm}^{2}$. The cells were incubated for two days before their use in the experiment.

For the initial generation of spheroids the monolayer cell culture was removed off the substrate after the four day incubation, using 0,25\% Trypsin-EDTA, and placed in nutrient medium with $5 \%$ carboxymethyl cellulose (Bio-Rad, USA) at concentration of $5 \times 10^{5}$ cells $/ \mathrm{ml}$. Then the plates were incubated on an orbital shaker (Orbital shaker, PSU-10i, Biosan, Latvia) at $50 \mathrm{rpm}$ for 3-5 hours. Half of culture medium was replenished every 3 days. A spheroid culture was maintained for 14 days. Further MCTS for immunohistochemical assay were obtained from paraffin blocks. Sections were cut from each block, dewaxed in xylene, and then hydrated using graded concentrations of ethanol in distilled water. Microwave antigen retrieval was performed before incubation with primary antibodies at room temperature for $5 \mathrm{~min}$. Samples were incubated with each antibody.

To evaluate the expression of EMT markers in monolayer culture cells were seeded on cover glass in 6-well plates (Flat bottom cell+, Sarstedt, USA) at a density of $2 \times 10^{4}$ cells $/ \mathrm{cm}^{2}$. Detection of markers in 2D and 3D cell culture was performed using immunohistochemistry method with primary monoclonal antibodies: Cytokeratin (clone AE1/AE3, Dako, USA), PanCytokeratin 5/6/8/18 (clone 5D3 and LP34, Dako, USA), ER $\alpha$ (clone EP1, Dako, USA), EGFR (Dako, USA), EpCAM (epithelial cell adhesion molecule), (Sigma, Sweden), p53 (tumor suppressor protein)(Sigma, Sweden). Visualization of proteins was performed according to the manufacturer's recommendations using the system PolyVue HRP/DAB Detection system (“DiagnosticBioSystem”, USA, № PV100D). Histological samples of the cells were photographed to compare the morphological characteristics and the expression of proteins in monolayer and spheroid culture (origin magnification $\times 100$, Cannon, Stemi 2000, Zeiss).

\section{Results of research}

As shown in Table 1, the significant difference was observed in the expression of tumor associated markers (ER, p53, vim, EpCAM, Cks and EGFR) in 2D and 3D cell cultures. Notably, a uniform diffusive distribution of markers was shown in 2D cell culture (Fig. 1-7, b). As apposed, in cell aggregates outer cell layers expressing these markers (Fig. 1-7, a).

Table 1

Expression of main markers of epithelial-mesenchymal transition in $2 \mathrm{D}$ and $3 \mathrm{D}$ cell culture (breast adenocarcinoma, MCF-7)

\begin{tabular}{|c|c|c|c|c|c|c|}
\hline \multirow[t]{2}{*}{ Markers } & \multicolumn{3}{|c|}{ 3D cell culture, $\%$ of cells } & \multicolumn{3}{|c|}{ 2D cell culture, $\%$ of cells } \\
\hline & “+” & “土” & “-” & “+” & “ $\pm ”$ & “_" \\
\hline EGFR & 10 & 15 & 75 & 75 & 20 & 5 \\
\hline EpCAM & 3 & 10 & 87 & 60 & 25 & 15 \\
\hline ER & 20 & 20 & 60 & 56 & 40 & 4 \\
\hline panCK & 30 & 15 & 55 & 95 & 5 & 0 \\
\hline Vim & 5 & 5 & 90 & 0 & 0 & 100 \\
\hline $\mathrm{p} 53$ & 60 & 30 & 10 & 50 & 45 & 5 \\
\hline AE1/AE3 & 5 & 10 & 85 & 100 & 0 & 0 \\
\hline
\end{tabular}



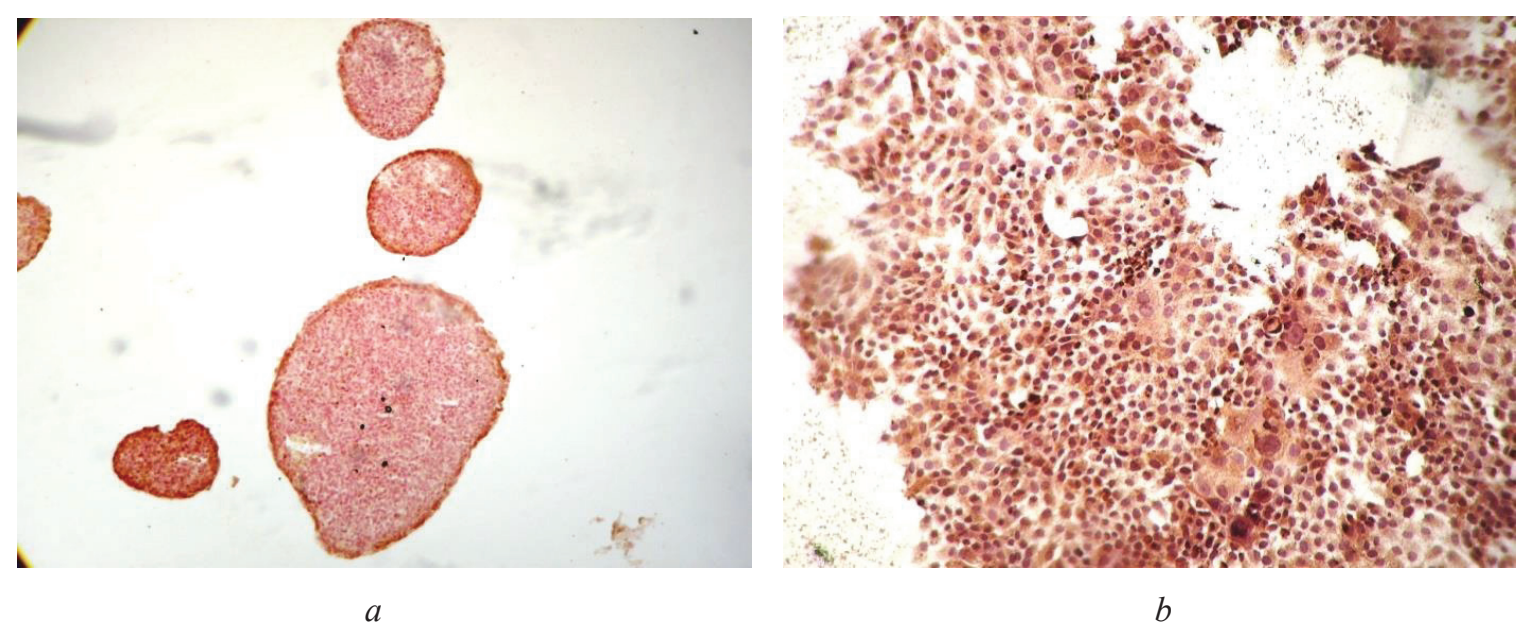

Fig. 1. EGFR expression in MCF-7 cell line, breast adenocarcinoma: $a-$ in $3 \mathrm{D}$ cell culture; $b-$ in $2 \mathrm{D}$ cell culture

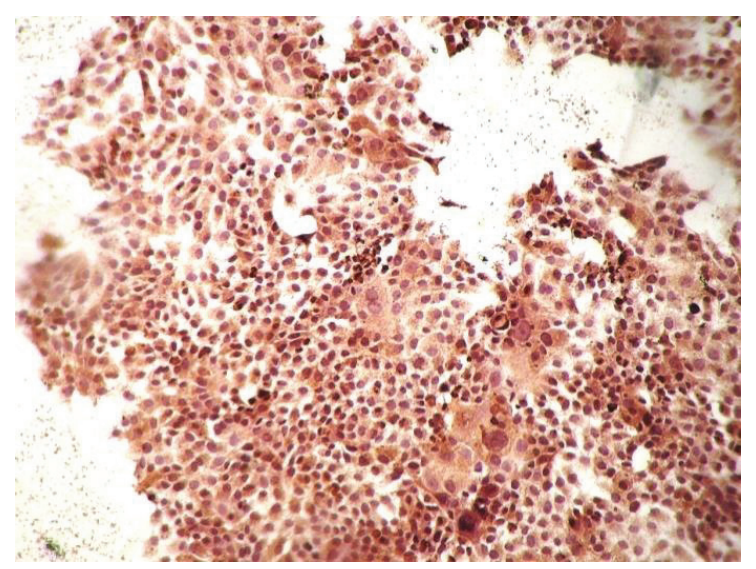

a

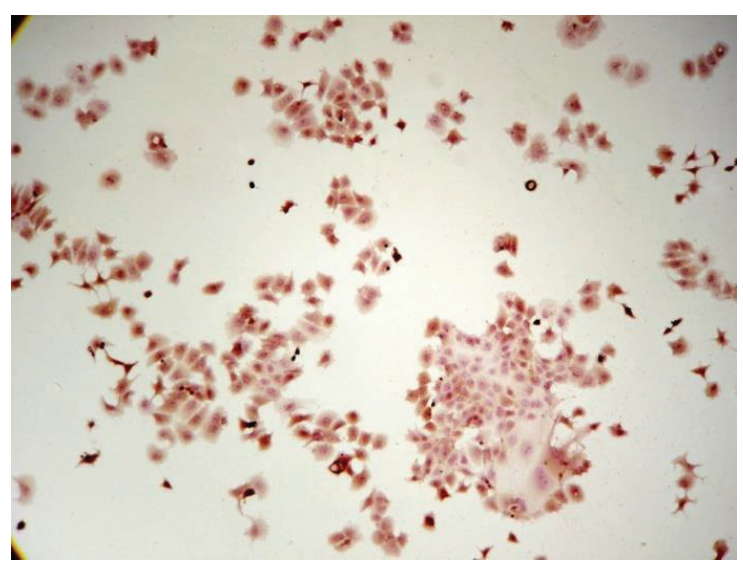

b

Fig. 2. EpCAM expression in MCF-7 cell line, breast adenocarcinoma: $a-$ in $3 \mathrm{D}$ cell culture; $b-$ in $2 \mathrm{D}$ cell culture

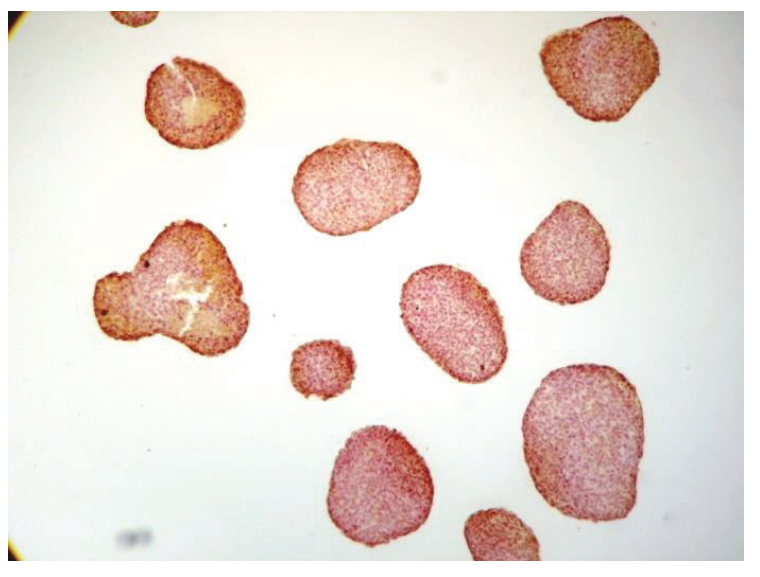

a

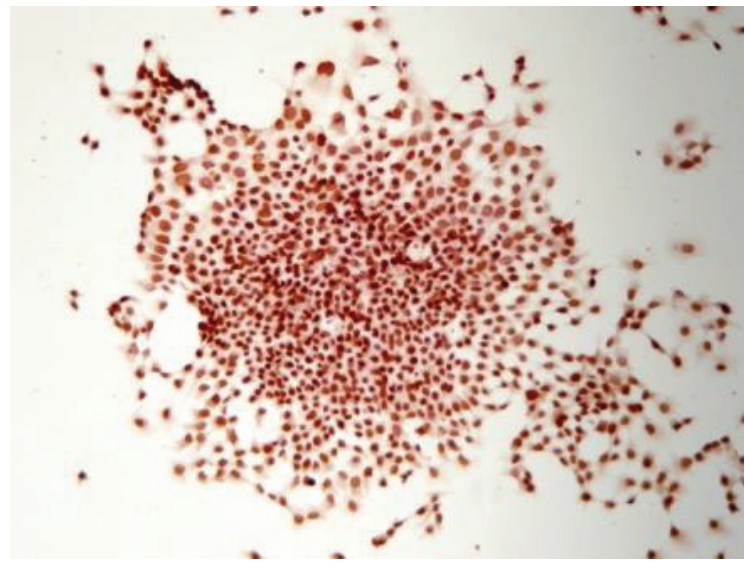

b

Fig. 3. ER expression in MCF-7 cell line, breast adenocarcinoma: $a-$ in $3 \mathrm{D}$ cell culture; $b-$ in $2 \mathrm{D}$ cell culture

According to literature data [9] actively proliferating cells are in the spheroid outer cell layers. Such cell distribution may be caused not only by the limited diffusion substances into the inner layers 
of the spheroid, but with the heterogeneity of the population and functional cellular composition. On the one hand increasing of the cell subpopulations number may be caused by EMT, but on the other hand it is caused by a small subpopulation of tumor stem cells, that closely related to invasion and metastasis [10].

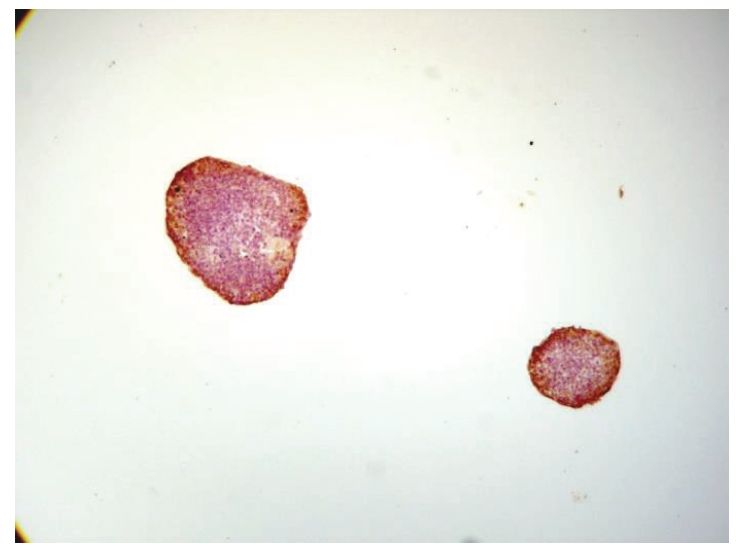

a

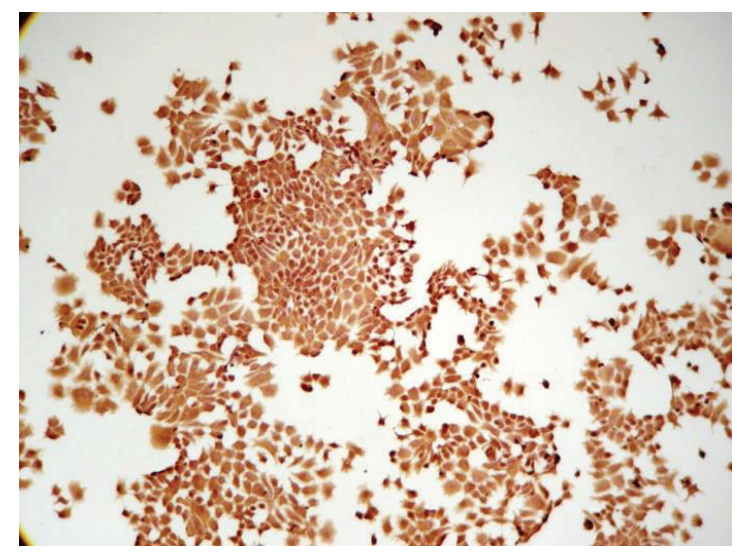

b

Fig. 4. Pan-CK expression in MCF-7 cell line, breast adenocarcinoma: $a-$ in $3 \mathrm{D}$ cell culture; $b-$ in $2 \mathrm{D}$ cell culture
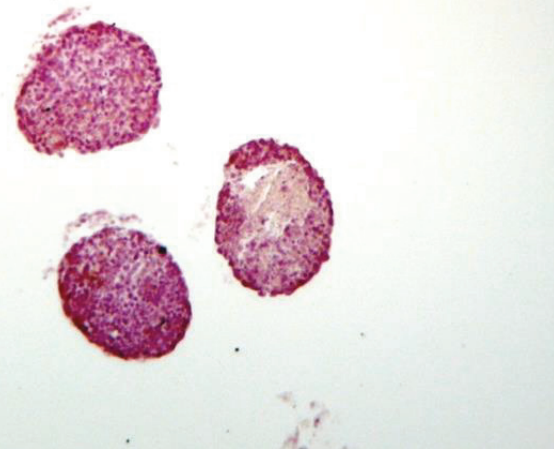

$a$

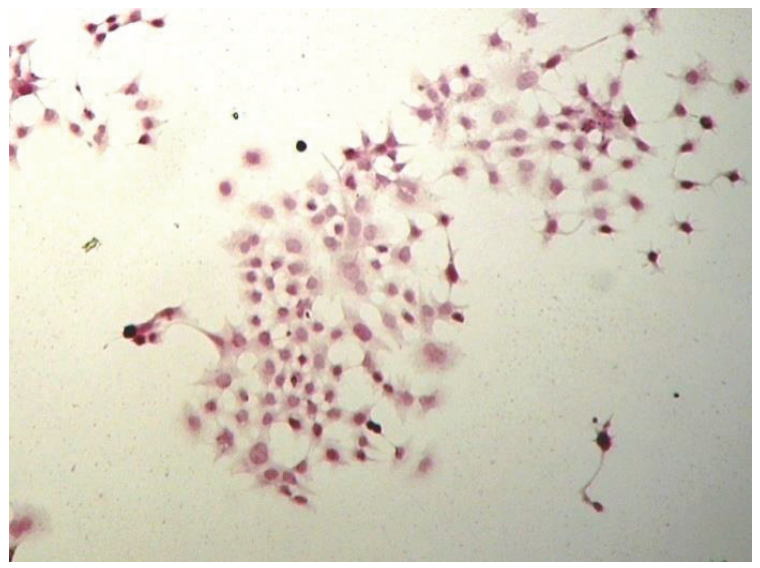

$b$

Fig. 5. Vim expression in MCF-7 cell line, breast adenocarcinoma: $a-$ in $3 \mathrm{D}$ cell culture; $b-$ in $2 \mathrm{D}$ cell culture

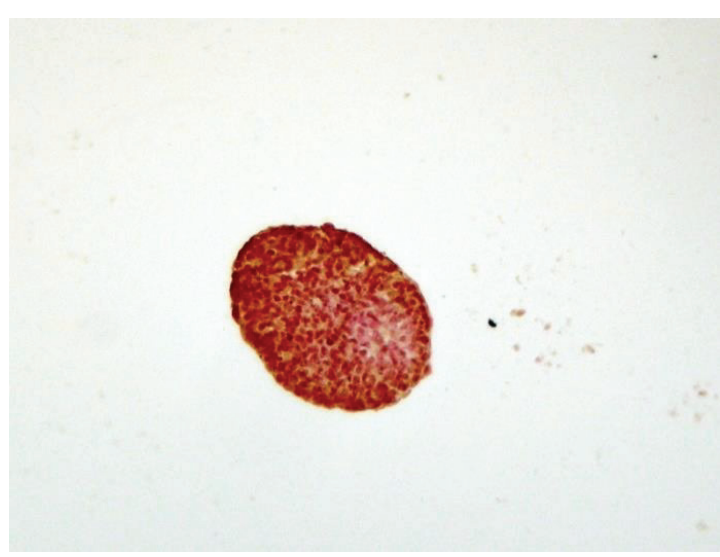

$a$

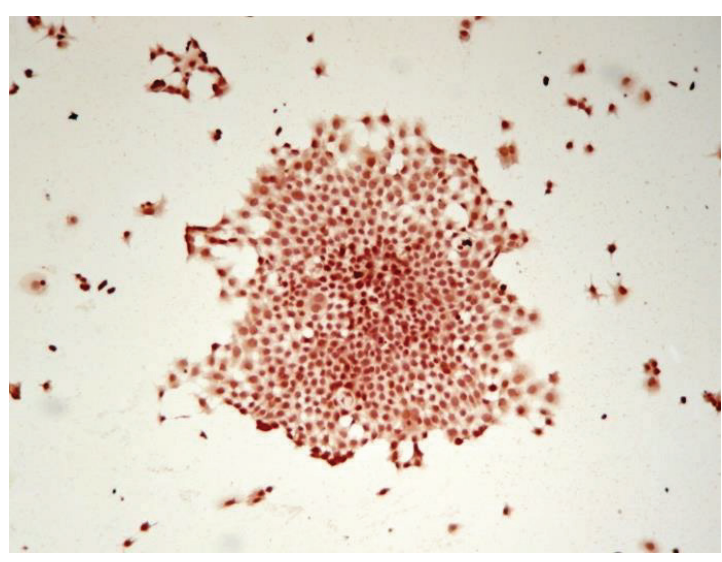

$b$

Fig. 6. P53 expression in MCF-7 cell line, breast adenocarcinoma: $a-$ in $3 \mathrm{D}$ cell culture; $b-$ in $2 \mathrm{D}$ cell culture 

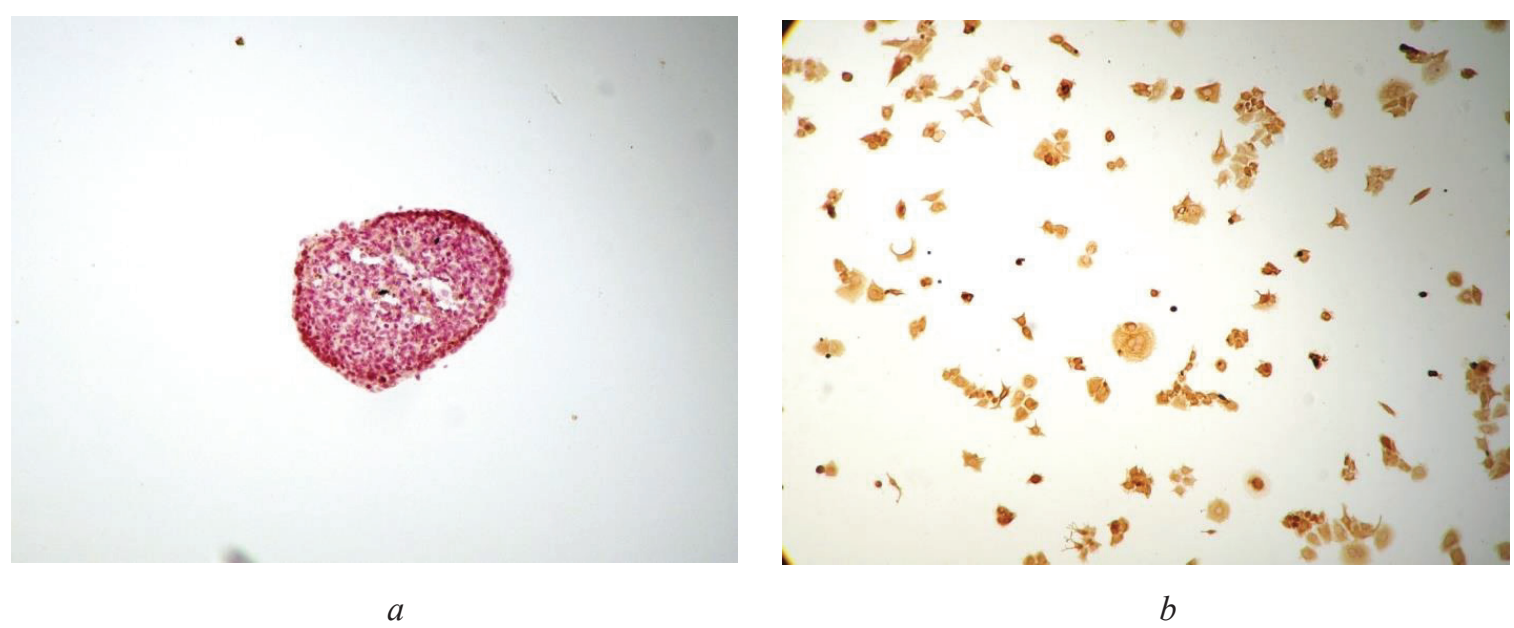

Fig. 7. AE1/AE3 expression in MCF-7 cell line, breast adenocarcinoma: $a-$ in $3 \mathrm{D}$ cell culture; $b-$ in $2 \mathrm{D}$ cell culture

As shown in Fig. 8, the percentage of marker positive cells (ER+, EGFR+, EpCAM+, panCK+, AE1/AE3+) in monolayer culture is 1.25-2 times more than in spheroid culture. Reduced of manifestation of tumor associated markers in 3D cell culture may indicate an increase of stem properties. In contrast, tumor spheroids consist of fewer cells with the expression of epithelial markers such as EpCAM and AE1/AE3, but they contain a large number of cells that expressed mesenchymal marker vimentin by $5 \%$ and and p53 by $10 \%$. This may indicate that the cells acquire a mesenchymal phenotype. However, tumor cells of monolayer cell culture were not expressed vimentin (Fig. 8). Unfortunately, we are not investigated all of mesenchymal markers of epithelial-mesenchymal transition, but it will be our next objective.

The results of our work are in good agreement with the literature data [11]. Therefore, our data showed that the $3 \mathrm{D}$ cell culture more than $2 \mathrm{D}$ cell culture characterized processes of epitelial-mesenchymal transition.

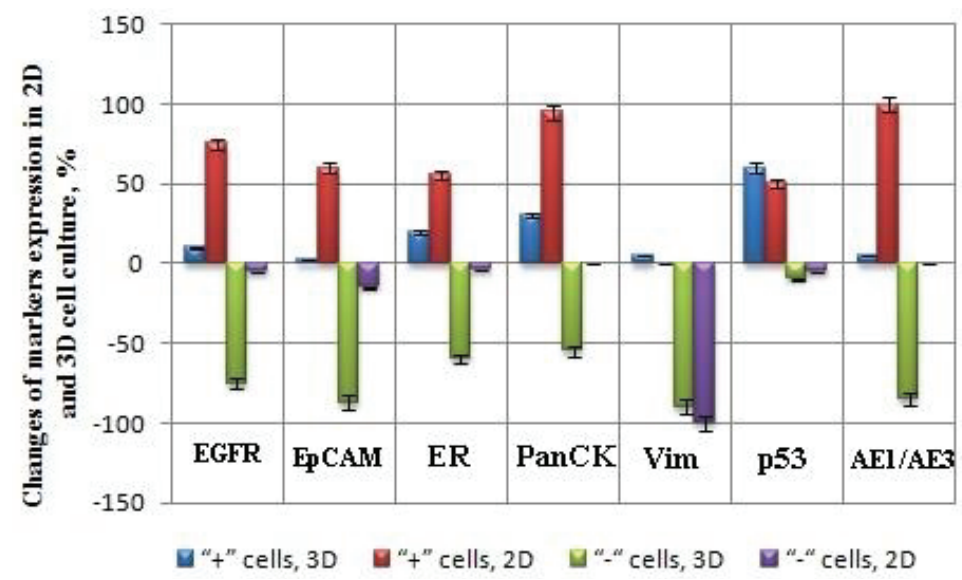

Fig. 8. Changes in the expression of markers of epithelial-mesenchymal transition in $2 \mathrm{D}$ and $3 \mathrm{D}$ cell culture MCF-7, \% “+” and “-” cells

\section{Discussion of results of research}

Originally, breast tumor derived from epithelial tissue, which is characterized by a multi-layer morphology with apical-basal polarity. However, the mesenchymal tissue has no polarity. At the same time in the mesenchymal cells increased the expression of mesenchymal associated molecules $\mathrm{N}$-cadherin, fibronectin and vimentin [12]. EMT closely related to EpCAM expression (epithelial cell adhesion molecule). Such tumor tissue as primary and metastatic breast cancer has high expression levels of EpCAM that is associated with poor prognosis, cell proliferation, migration and 
possibly the initiation of cancer [13]. EpCAM expression modulation is a way to influence the oncogenic potential of tumor cells for certain types of tumors [14]. EpCAM has been recently identified as an additional marker for tumor-associated stem cells. Cancer cells that express EpCAM have greater oncogenic potential than EpCAM-negative cells. Vimentin is another important molecule of mesenchymal origin, which takes part in the EMT. This molecule is found in epithelial cells involved in the physiological or pathological processes that require migration of epithelial cells [15]. Vimentin's overexpression in cancer correlates well with accelerated tumor growth, invasion, and poor prognosis. Although EMT is associated with several tumorigenic events, vimentin's role in the underlying events mediating these processes remains unknown. By virtue of its overexpression in cancer and its association with tumor growth and metastasis, vimentin serves as an attractive potential target for cancer therapy [16]. Consequently, EMT is caused by a wide range of molecular processes. One of these processes have the binding of growth factors such as EGF (epidermal growth factor), with a specific receptor (EGFR) on the cell surface. Thus, such binding leads to the receptor activation and stimulation of EMT in breast tumor cells [17]. Thereby, it changes the expression of vimentin and E-cadherin [18]. Scientists have shown that inhibition of EGFR expression leads to the suppression of EMT and reduction ability, migration and invasion of tumor cells [19]. In contrast, EGFR activation increases the expression of vimentin (mesenchymal marker) and reduces the expression of pan-cytokeratins (epithelial markers) [20]. Recent studies have shown that an important role in the regulation of EMT plays proapoptotic protein p53. It is a transcription factor and it regulates expression of genes that promote apoptosis, aging and cell cycle arrest [21]. The ability of p53 to regulate metastasis, migration and invasion occurs by modulating of EMT and suppression of the basic properties of cancer stem cells (CSC) [22].

\section{Conclusion}

In this study we compared the expression of markers of epithelial-mesenchymal transition in monolayer and spheroid cell culture of breast adenocarcinoma. The results showed differences in the expression of EMT markers in 2D and 3D culture. Thus, the percentage of epithelial markers (Cks and EpCAM) in tumor spheroids is less than in cells of monolayer however spheroids cells begin expressing a mesenchymal marker - vimentin. In 3D cell culture only the outer cell layers expressed EMP proteins unlike 2D cell culture in which all of cells showed equally expression. It is evident, that these two cell cultures have differences in marker expression, and, as we have shown previously [23], in morphology, proliferation, and response to anticancer drugs.

\section{References}

[1] Khaitan, D., Dwarakanath, B. S. (2006). Multicellular spheroids as an in vitro model in experimental oncology: applications in translational medicine . Expert Opinion on Drug Discovery, 1 (7), $663-675$. doi: $10.1517 / 17460441.1 .7 .663$

[2] Hirschhaeuser, F., Menne, H., Dittfeld, C., West, J., Mueller-Klieser, W., Kunz-Schughart, L. A. (2010). Multicellular tumor spheroids: An underestimated tool is catching up again. Journal of Biotechnology, 148 (1), 3-15. doi: 10.1016/j.jbiotec.2010.01.012

[3] Yamada, K. M., Cukierman, E. (2007). Modeling Tissue Morphogenesis and Cancer in 3D. Cell, 130 (4), 601-610. doi: 10.1016/j.cell.2007.08.006

[4] Gurski, L., Petrelli, N., Jia, X., Farach-Carson, M. (2010). 3D matrices for Anticancer Drug Testing and Development. Oncology Issues, 25, 20-25

[5] Dufau, I., Frongia, C., Sicard, F., Dedieu, L., Cordelier, P., Ausseil, F. et. al. (2012). Multicellular tumor spheroid model to evaluate spatio-temporal dynamics effect of chemotherapeutics: application to the gemcitabine/CHK1 inhibitor combination in pancreatic cancer. BMC Cancer, 12 (1). doi: 10.1186/1471-2407-12-15

[6] Marcucci, F., Stassi, G., De Maria, R. (2016). Epithelial-mesenchymal transition: a new target in anticancer drug discovery. Nature Reviews Drug Discovery, 15 (5), 311-325. doi: 10.1038/nrd.2015.13

[7] Thiery, J. P., Lim, C. T. (2013). Tumor Dissemination: An EMT Affair. Cancer Cell, 23 (3), 272-273. doi: 10.1016/j.ccr.2013.03.004 
[8] Bill, R., Christofori, G. (2015). The relevance of EMT in breast cancer metastasis: Correlation or causality? FEBS Letters, 589 (14), 1577-1587. doi: 10.1016/j.febslet.2015.05.002

[9] Edmondson, R., Broglie, J. J., Adcock, A. F., Yang, L. (2014). Three-Dimensional Cell Culture Systems and Their Applications in Drug Discovery and Cell-Based Biosensors. ASSAY and Drug Development Technologies, 12 (4), 207-218. doi: 10.1089/adt.2014.573

[10] Dave, B., Mittal, V., Tan, N. M., Chang, J. C. (2011). Epithelial-mesenchymal transition, cancer stem cells and treatment resistance. Breast Cancer Research, 14 (1), 202. doi: 10.1186/bcr2938

[11] Lindsey, S., Langhans, S. A. (2014). Crosstalk of Oncogenic Signaling Pathways during epithelial-mesenchymal transition. Transition. Frontiers in Oncology, 4. doi: 10.3389/fonc.2014.00358

[12] Lamouille, S., Xu, J., Derynck, R. (2014). Molecular mechanisms of epithelial-mesenchymal transition. Nature Reviews Molecular Cell Biology, 15 (3), 178-196. doi: 10.1038/nrm3758

[13] Gostner, J. M., Fong, D., Wrulich, O. A., Lehne, F., Zitt, M., Hermann, M. et. al. (2011). Effects of EpCAM overexpression on human breast cancer cell lines. BMC Cancer, 11 (1). doi: 10.1186/1471-2407-11-45

[14] Spizzo, G., Fong, D., Wurm, M., Ensinger, C., Obrist, P., Hofer, C. et. al. (2011). EpCAM expression in primary tumour tissues and metastases: an immunohistochemical analysis. Journal of Clinical Pathology, 64 (5), 415-420. doi: 10.1136/jcp.2011.090274

[15] Gires, O., Klein, C. A., Baeuerle, P. A. (2009). On the abundance of EpCAM on cancer stem cells. Nature Reviews Cancer, 9 (2), 143-143. doi: 10.1038/nrc2499-c1

[16] Kalluri, R., Weinberg, R. A. (2009). The basics of epithelial-mesenchymal transition. Journal of Clinical Investigation, 119 (6), 1420-1428. doi: 10.1172/jci39104

[17] Hardy, K. M., Booth, B. W., Hendrix, M. J. C., Salomon, D. S., Strizzi, L. (2010). ErbB/EGF Signaling and EMT in Mammary Development and Breast Cancer. Journal of Mammary Gland Biology and Neoplasia, 15 (2), 191-199. doi: 10.1007/s10911-010-9172-2

[18] Misra, A., Pandey, C., Sze, S. K., Thanabalu, T. (2012). Hypoxia Activated EGFR Signaling Induces Epithelial to Mesenchymal Transition (EMT). PLoS ONE, 7 (11), e49766. doi: 10.1371/journal.pone.0049766

[19] Chang, Z.-G., Wei, J.-M., Qin, C.-F., Hao, K., Tian, X.-D., Xie, K. et. al. (2012). Suppression of the Epidermal Growth Factor Receptor Inhibits Epithelial-Mesenchymal Transition in Human Pancreatic Cancer PANC-1 Cells. Digestive Diseases and Sciences, 57 (5), 1181-1189. doi: 10.1007/s10620-012-2036-4

[20] Serrano, M. J., Ortega, F. G., Alvarez-Cubero, M. J., Nadal, R., Sanchez-Rovira, P., Salido, M. et. al. (2014). EMT and EGFR in CTCs cytokeratin negative non-metastatic breast cancer. Oncotarget, 5 (17), 7486-7497. doi: 10.18632/oncotarget.2217

[21] Goh, A. M., Coffill, C. R., Lane, D. P. (2010). The role of mutant p53 in human cancer. The Journal of Pathology, 223 (2), 116-126. doi: 10.1002/path.2784

[22] Chang, C.-J., Chao, C.-H., Xia, W., Yang, J.-Y., Xiong, Y., Li, C.-W. et. al. (2011). p53 regulates epithelial-mesenchymal transition and stem cell properties through modulating miRNAs. Nature Cell Biology, 13 (3), 317-323. doi: 10.1038/ncb2173

[23] Gergeliuk, T., Perepelytsina, O., Sidorenko, M. (2014). Comparing the impact of conditioned medium from human mesenchymal stem cells and interferon-population and the development of cancer cells MCF-7. Bukovina Medical Journal, 18 (2), 18-23. 